\title{
MODELAGEM E ANÁLISE DA CONFIABILIDADE DE UM SISTEMA COMPLEXO DE UMA AERONAVE QUADRIMOTORA
}

Rafael Kayque dos Santos (rafaelkayquepeixoto@gmail.com) - Universidade de Brasília - Faculdade do Gama

Pedro Henrique Andreoli Luminati (ph.luminati@hotmail.com) - Universidade de Brasília - Faculdade do Gama

Jonatas Ribeiro Mangabeiro (jonatas.ribeiro.mangabeiro@gmail.com) - Universidade de Brasília Faculdade do Gama

Viviane Vasconcellos Ferreira Grubisic (vivianegrubisic@unb.br) - Universidade de Brasília Faculdade de Tecnologia

\section{RESUMO}

Em vista de garantir a segurança e o alto desempenho de uma aeronave em voo, é necessário assegurar o funcionamento de seus múltiplos subsistemas integrados. Nesse seguimento, as aeronaves contam com diversas redundâncias que permitem seus subsistemas continuar operando, mesmo com a ocorrência de falha em alguns de seus componentes. Para tanto, é de grande interesse da engenharia assegurar um alto nível de confiabilidade associada ao funcionamento de cada um desses subsistemas. No presente artigo, foi abordado um estudo de confiabilidade de um sistema complexo formado pela integração dos conjuntos de motores e controles de uma aeronave quadrimotora. Para tal, por meio da implementação de algoritmos matemáticos, as etapas obtidas envolveram o cálculo de confiabilidade do sistema considerado, identificação dos componentes mais críticos, estudo dos modos e efeitos de falha por meio da FMEA, e estudo das causas raízes, realizado por meio da FTA.

Palavras chave: confiabilidade; criticidade; análise de falha; sistema complexo; aeronave quadrimotora; 


\section{INTRODUÇÃ̃}

O estudo da confiabilidade é historicamente decorrente da necessidade de se comparar aviões no que diz respeito ao número de acidentes ocorridos por hora de voo, é o que propõe Knight (1991).

Leemis (1995, p. 384) define confiabilidade como sendo "a probabilidade do item de desempenhar adequadamente o seu propósito especificado, por um determinado período de tempo e sob condições predeterminadas", de acordo com as especificações de projeto do produto. Um aumento da confiabilidade de um sistema está intimamente ligado a uma menor probabilidade de ocorrência de falhas do mesmo. De igual modo, Fogliatto (2006) propõe que uma confiabilidade maior, promove um nível de qualidade superior de manufatura, o que, por sua vez, fomenta uma maior aceitação da unidade produzida pelo mercado.

Uma unidade de aeronave de um dado fabricante, por exemplo, pode operar sob condições ambientais extremas, realizando muitos voos ao dia, o que acarreta em muitas decolagens e aterrisagens ao dia. Já outra unidade do mesmo fabricante, pode operar seus voos sob condições ambientais mais suaves, percorrendo rotas mais longas e realizando menos decolagens e aterrisagens ao dia (DODSON, 2006). Esta alta variabilidade das condições de contorno de operação da aeronave, requisita que a aeronave possua um alto índice de confiabilidade associada aos seus subsistemas integrados. Neste sentido, as pesquisas sobre confiabilidade começaram a desenvolver-se a fim de se tornarem cada vez mais aplicáveis em projetos de sistemas de engenharia que requisitam de alta robustez como é o caso da indústria aeronáutica.

Para efeito de análise de confiabilidade, no presente artigo, será abordado um conjunto de subsistemas críticos de um modelo de aeronave exposto por Pettit e Turnbull (2001) e Lenz e Rhodin (2011). Para tanto, será utilizado o software Proconf (software orientado para análise de confiabilidade), de maneira a auxiliar na obtenção de gráficos relacionados às medidas de confiabilidade. Não obstante, será apresentado o procedimento de implementação de cálculo da estimativa da confiabilidade do sistema total considerado, tendo como base o método do tieset. Também será calculada a medida de Importância de componentes de Birnbaum, para orientar na detecção de componentes críticos relacionados aos subsistemas da aeronave apontados. O estudo dos modos e efeitos de falha do sistema será realizado por meio da análise da FMEA (Análise dos Modos e Efeitos de Falha). As causas raízes serão estudadas pela FTA 
(Análise de Árvore de Falhas), a partir do levantamento de uma combinação de eventos que caracterizam uma possível falha potencial do sistema.

\section{FUNDAMENTOS DE CONFIABILIDADE}

O estudo da confiabilidade é realizado implementando funções matemáticas que modelam o comportamento de variáveis aleatórias. Para tanto, se faz necessário inserir o conceito de variável aleatória. Segundo Pinheiro (2013, p.50), "variável aleatória (v.a.) é uma função que associa a cada elemento de um espaço amostral um número real". As variáveis aleatórias podem ser discretas ou contínuas. Neste trabalho, a variável aleatória considerada é do tipo contínua, e expressa o tempo de operação da aeronave.

No estudo de confiabilidade, quatro funções são utilizadas para caracterizar o comportamento de variáveis aleatórias:

- Função de densidade de probabilidade ou $\mathrm{f}(\mathrm{t})$;

- Função de distribuição cumulativa ou $\mathrm{F}(\mathrm{t})$;

- Função de confiabilidade ou R(t);

- Função de risco ou h(t);

A partir do cálculo de uma delas, as demais variáveis podem ser derivadas por manipulação matemática (DODSON, 2006).

Para efeito de estudo, no presente artigo serão utilizadas as medidas de $\mathrm{R}(\mathrm{t})$ e $\mathrm{F}(\mathrm{t})$. A função de confiabilidade $\mathrm{R}(\mathrm{t})$ expressa a probabilidade de sobrevivência da unidade por um determinado tempo de operação "t", podendo ser exibido em anos, meses, horas, ciclos etc. Como $\mathrm{R}(\mathrm{t})$ é uma probabilidade, o domínio dos valores obtidos está entre 0 e 1 . Quanto maior for o valor de tempo de operação da unidade, menor tende a ser o valor de $\mathrm{R}(\mathrm{t})$ associado, de modo que os valores de $\mathrm{R}(\mathrm{t})$ diminuem de 1 para 0 à medida que $t$ aumenta. Matematicamente, no domínio contínuo, $\mathrm{R}(\mathrm{t})$ é representada pela equação (1) como sendo a integral da função de densidade de probabilidade $\mathrm{f}(\mathrm{t})$ (DODSON, 2006).

$$
R(t)=\int_{t}^{\infty} f(t) d t
$$


A função acumulada de probabilidade $(\mathrm{F}(\mathrm{t}))$ é o complemento da função de confiabilidade, conhecida também como função de não confiabilidade. Nesse sentido, $F(t)$ representa a probabilidade de falha da unidade que está em operação. Logo, as mesmas análises matemáticas relacionadas a $\mathrm{R}(\mathrm{t})$, podem ser utilizadas para $\mathrm{F}(\mathrm{t})$ : quanto menor for o valor de $t$, menor tende a ser o valor de $\mathrm{F}(\mathrm{t})$ associado, exibindo valores que partem de 0 para 1 , à medida que $t$ aumenta. Nesse sentido, Dodson (2006) relaciona as duas medidas de confiabilidade, através da expressão matemática representada pela equação (2) que pode ser expressa como:

$$
F(t)+R(t)=1
$$

Onde,

$$
F(t)=\int_{0}^{t} f(t) d t
$$

\subsection{Estimativa de confiabilidade de sistemas}

Arranjos estruturais de um sistema de engenharia podem ser simples ou complexos. Fogliatto (2006) expõe métodos utilizados para determinação de confiabilidade de sistemas simples (série e paralelo, combinações de série-paralelo e arranjos do tipo k-em-n). Métodos para cálculos de confiabilidade de sistemas complexos são apresentados ainda pelo autor, sendo estes: (i) método da decomposição, (ii) métodos do tie set e cut set, (iii) método da tabela booleana, e (iv) método da redução. Para efeito de estudo, este presente trabalho se propõe a utilizar o método do tie set para o cálculo de confiabilidade do sistema total considerado $\mathrm{R}_{\mathrm{s}}$.

\subsection{Método do tie set}

O método do tie set busca determinar a confiabilidade total de um sistema complexo, por meio do seguinte raciocínio: é necessário identificar os caminhos mínimos de operação que conduzam ao funcionamento do sistema. Esses caminhos são chamados de tie set's mínimos. A confiabilidade de um sistema complexo, seja qual ele for, é dada pela união de todos os tie set’s mínimos (FOGLIATTO, 2006). 


\subsection{Medidas de importância de componentes}

Høyland (2003) demonstra que a importância de um componente depende de dois fatores: a localização do componente no sistema e a confiabilidade do componente em questão. As medidas de importância podem ser usadas como um método de detecção dos possíveis pontos fracos do sistema, tornando possível a implementação de correções e melhorias no projeto de produto ou processo analisado. A partir da identificação dos componentes críticos do sistema, a confiabilidade total pode ser melhorada ao se adicionar, por exemplo, um componente de alta confiabilidade, introduzir componentes redundantes no sistema, melhorar a manutenibilidade do componente etc. (ELSAYED, 1996).

Quatro medidas de importância são apresentadas por Fogliatto (2006), sendo elas: (i) Medida de Birnbaum, (ii) Importância crítica, (iii) Vesely-Fussell e (iv) Potencial de melhoria. Para efeito de estudo, o presente trabalho se propõe a utilizar a medida de Birnbaum.

\subsection{Medida de Importância de Birnbaum}

De acordo com a medida de Importância de Birnbaum, o componente mais fraco de um arranjo em série é o mais importante. Desse modo, o componente de menor confiabilidade é apontado como o mais importante. Já em um sistema em paralelo, o componente de maior confiabilidade é crítico. Para um sistema complexo, a análise de criticidade é feita tanto em termos da confiabilidade do componente, quanto em termos da posição que o mesmo ocupa no sistema (FOGLIATTO, 2006).

Høyland (2003) apresenta matematicamente por meio da equação (4) a medida de importância de Birnbaum, em termos da derivada parcial da confiabilidade total do sistema $\left(R_{\mathrm{s}}\right)$ em um tempo $t$, em relação a derivada parcial do componente $i$ que é crítico para o sistema.

$$
I^{B}\left(\frac{i}{t}\right)=\frac{\partial R s(r(t))}{\partial R i(t)}
$$

\subsection{FMEA}

A FMEA (Failure Mode and Effects Analysis ou Análise dos Modos e Efeitos de Falha) trata de um método sistemático de análise de falhas, realizado por uma equipe 
multidisciplinar de engenheiros especialistas. Essa análise visa (i) identificar e analisar os modos e efeitos de falhas que podem surgir em um produto ou processo; indicar ações práticas que: (ii) eliminem ou reduzam a chance de ocorrência dessas falhas; (iii) que aumentem a chance de detecção dos modos de falha; (iv) que diminuam o grau de severidade dos efeitos de falha; (v) além de possibilitar o registro do estudo realizado, criando um referencial técnico que colabore em atualizações e criações futuras do projeto de produto e processo (FOGLIATTO, 2006). O presente trabalho expõe adiante nos resultados (seção 4), a construção da FMEA para o sistema da aeronave considerado.

\subsection{Análise de árvore de falhas (FTA)}

Smith (2001) propõe uma definição para a Árvore de Falhas ou FTA (Fault Tree Analysis) como sendo um método gráfico que descreve a combinação de eventos com o objetivo de caracterizar a falha de um determinado sistema. A falha no sistema é denominada de evento topo e as suas ramificações são as possíveis causas de sua falha, onde operadores lógicos são empregados para determinar a propagação da falha.

Tais operadores lógicos utilizados são o "E" e "OU", onde para o caso do operador "E" todas as entradas necessitam de ocorrer para que a saída ocorra, sendo que para o operador "OU", qualquer entrada faz com que a saída ocorra. Desta forma, Flogiatto (2006) afirma que o operador lógico "E" representa um sistema em paralelo que é caracterizado por uma condição mais segura e o operador lógico "OU" representa a situação de menor segurança que corresponde um sistema em série. Birollini (2007) acrescenta que a FTA pode ser aplicada juntamente com a FMEA de forma a potencializar o estudo, visto que análise de árvore de falhas ajuda a identificar as relações lógicas existentes entre as causas e suas consequências.

\section{FUNDAMENTOS DA AERONAVE}

\subsection{Sistema de motores}

Para efeito de análise, o presente trabalho propõe uma divisão do sistema de motores da aeronave em quatro subsistemas, os quais dispõem de seus respectivos componentes. Tais subsistemas são: (i) motor (ii) aquecimento e resfriamento, (iii) sistema de combustível e (iv) hélice. As divisões dos sistemas de motores e controles em subsistemas e componentes, 
propostas pelo presente trabalho, estão em conformidade com o modelo de aeronave apresentado por Pettit e Turnbull (2001).

Para Pettit e Turnbull (2001), o subsistema do motor envolve todos os componentes do cofre do motor bem como do sistema de exaustão. Ainda segundo os autores, o subsistema de combustível da aeronave inclui os componentes que trabalham com o objetivo de fornecer a quantidade adequada de combustível aos motores, em qualquer regime de operação e altitude de voo. Já a bomba injetora de combustível, apesar de ser acionada pelo motor, faz parte do subsistema de combustível. Não obstante, o tanque e as linhas de combustível também estão inclusos neste último. O subsistema de aquecimento e resfriamento (AeR) da aeronave engloba todos os componentes responsáveis pela tarefa de controlar a temperatura e os fluxos de ar nas cabines do piloto e passageiros. Finalmente, o subsistema da hélice, de igual modo, inclui componentes envolvidos na conversão do torque do motor em empuxo para a aeronave.

Por meio de análise de Importância de Birnbaum, o subsistema de injeção de combustível foi detectado como ocupando o primeiro lugar no ranking de componentes mais críticos da aeronave. O segundo componente mais crítico do sistema de motores da aeronave, se trata do subsistema de aquecimento e resfriamento (ver subseção 4.2).

\subsection{Sistema de controles}

De igual modo, Pettit e Turnbull (2001), dividem o sistema de controles em nove subsistemas, que juntos recebem a missão de garantir o controle total da aeronave nos três eixos (x, y e z) de rotação durante o voo. Especificamente, são exibidos o controle direcional, controle longitudinal, controle lateral, flaps, estabilizadores (também chamados de compensadores), componentes hidráulicos (circuito hidráulico), trem de pouso, leme de direção e, por fim, as duas asas da aeronave. Os mecanismos de funcionamento de tais componentes não são abordados neste texto, mas estão disponíveis em Pettit e Turnbull (2001).

Por meio de análise de Importância de Birnbaum (ver seção 4.2), os estabilizadores foram detectados ocupando o primeiro lugar no ranking de componentes mais críticos da aeronave, tendo em vista o sistema de controles da aeronave. Eles têm por função desempenhar o papel de facilitar a pilotagem da aeronave e, na maioria dos aviões, ajudam a manter a direção 
da aeronave nos três eixos de rotação. O segundo componente mais crítico do sistema de controles da aeronave em questão são os flaps.

\subsection{Função Global}

Na FIGURA 1 pode-se observar a função global do sistema e como cada um dos subsistemas se relacionam entre si. Analisando da esquerda para a direita, nota-se a presença de um sistema complexo composto por quatro conjuntos de motores (por se tratar de um avião quadrimotor), seguido de um sistema em série responsável pelo controle da aeronave.

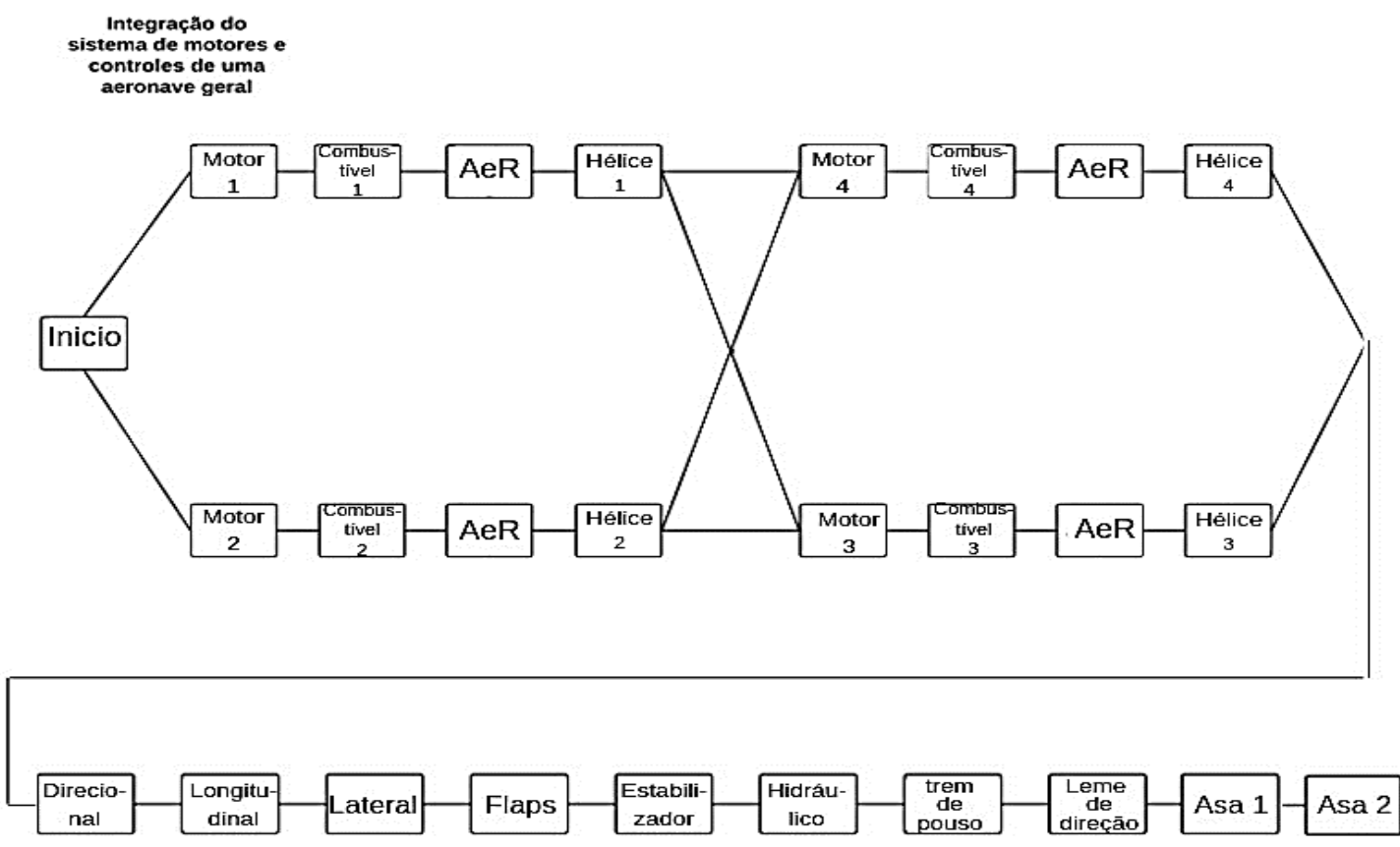

FIGURA 1 - Função global do sistema

FONTE: Elaborada pelos autores

No sistema complexo, os conjuntos dos motores estão conectados por uma ligação cruzada, o que, por sua vez, cria uma redundância no sistema. Essa redundância torna todo o sistema mais confiável devido ao fato de nenhum motor vir a falhar em decorrência da falha de outro motor. 


\section{RESULTADOS E DISCUSSÃO}

\subsection{Cálculo da confiabilidade do sistema $R_{\mathrm{s}}$}

Com base nos dados de confiabilidade dos componentes da aeronave exibidos por Pettit e Turnbull (2001) bem como por Lenz e Rhodin (2011), é possível calcular a confiabilidade total do sistema $\left(\mathrm{R}_{\mathrm{s}}\right)$. Para efeito de cálculo, foi considerado o sistema complexo formado pela integração dos sistemas de motores e controles da aeronave apresentada. Os dados utilizados para o cálculo de $\mathrm{R}_{\mathrm{S}}$ são mostrados na TABELA 1, onde $t$ expressa um tempo de missão de seis (6) horas de voo.

TABELA 1 - Dados de confiabilidade de componentes da aeronave.

FONTE: PETTIT e TURNBULL, 2001, p. 24-25.

\begin{tabular}{|c|c|c|c|}
\hline Componente & Confiabilidade & Componente & Confiabilidade \\
\hline $\mathrm{R}(\mathrm{t})$ motor & 0,99997436 & $\mathrm{R}(\mathrm{t})_{\text {longitudinal }}$ & 0,9999716 \\
\hline $\mathrm{R}(\mathrm{t})$ combustível & 0,99994005 & $\mathrm{R}(\mathrm{t})$ lateral & 0,9999998 \\
\hline $\begin{array}{c}\mathrm{R}(\mathrm{t}) \text { aquecimento } \\
\text { e resfriamento } \\
(\mathrm{AeR})\end{array}$ & 0,99997182 & $\mathrm{R}(\mathrm{t})_{\text {flaps }}$ & 0,9979040 \\
\hline $\mathrm{R}(\mathrm{t})$ hélice & 0,99997219 & $\mathrm{R}(\mathrm{t})$ estabilizador & 0,9884144 \\
\hline $\mathrm{R}(\mathrm{t})$ direcional & 0,9999956 & $\mathrm{R}(\mathrm{t})_{\text {hidráulico }}$ & 0,9993927 \\
\hline $\begin{array}{l}\mathrm{R}(\mathrm{t})_{\text {trem de }} \\
\text { pouso }\end{array}$ & 0,9966088 & $\begin{array}{l}\mathrm{R}(\mathrm{t})_{\text {leme de }} \\
\text { direção }\end{array}$ & 0,9999780 \\
\hline $\mathrm{R}(\mathrm{t})$ asa & 0,9999208 & & \\
\hline
\end{tabular}

Parâmetros da função densidade de probabilidade Weibull associada a dados de falha dos componentes pertencentes aos conjuntos de motores e controles da aeronave foram 
disponibilizados por Lenz e Rhodin (2011), onde $\alpha$ e $\beta$ representam, respectivamente, os parâmetros de escala e de forma da função em questão, mostrados na TABELA 2. Esses dados foram utilizados como entrada no software Proconf para a obtenção dos gráficos associados as medidas de confiabilidade, mostrados pela FIGURA 2 e FIGURA 3. Para efeito de simplificação, foi considerado apenas o componente do estabilizador, próprio do sistema de controles da aeronave, para a demonstração gráfica das medidas de $\mathrm{F}(\mathrm{t})$ e $\mathrm{R}(\mathrm{t})$.

TABELA 2 - Parâmetros da função densidade de probabilidade Weibull.

FONTE: LENZ e RHODIN, 2011, p. 44

\begin{tabular}{|c|c|c|c|c|c|}
\hline Componente & $\boldsymbol{\beta}$ & $\boldsymbol{\alpha}$ & Componente & $\boldsymbol{\beta}$ & $\boldsymbol{\alpha}$ \\
\hline motor & 1,58 & 4830 & flaps & 0,95 & 3956 \\
\hline combustível & 1,44 & 5130 & estabilizador & 0,73 & 2672,1 \\
\hline $\begin{array}{c}\text { hélice } \\
\text { e }\end{array}$ & 1,63 & 3740 & hidráulico & 1,14 & 3977,39 \\
\hline $\begin{array}{c}\text { aquecimento } \\
\text { (Aesfriamento }\end{array}$ & 1,60 & 4190 & de & 0,92 & 2895,62 \\
\hline direcional & 1,85 & 4729,02 & $\begin{array}{c}\text { pouso } \\
\text { direção }\end{array}$ & 1,65 & 3994,78 \\
\hline $\begin{array}{c}\text { lateral } \\
\text { longitudinal }\end{array}$ & 1,57 & 4718,22 & asa & 1,79 & 4250 \\
\hline
\end{tabular}




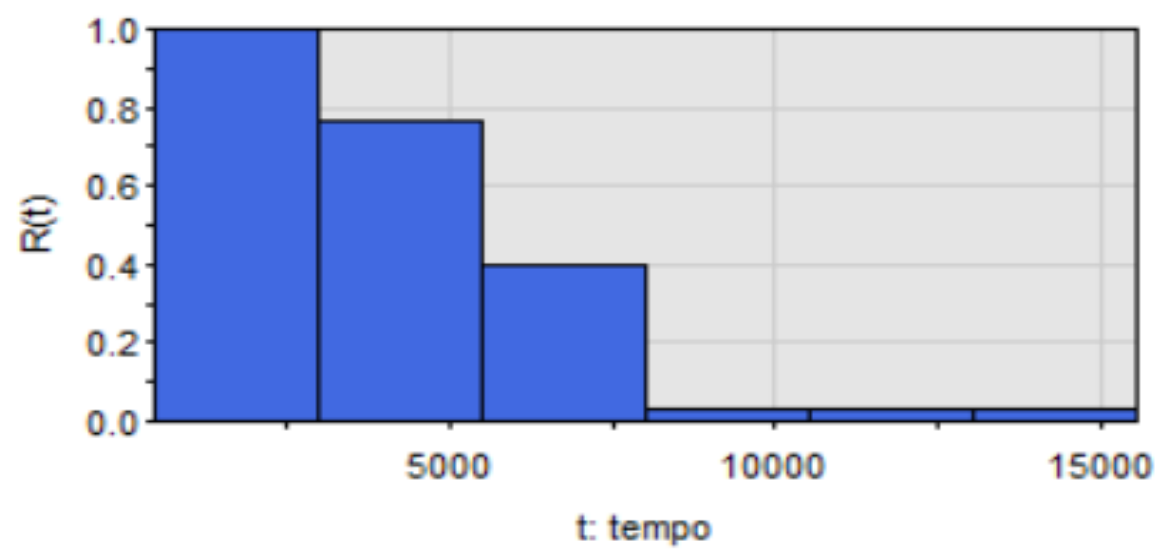

FIGURA 2 - Gráfico de barras de R(t) do estabilizador da aeronave FONTE: Proconf (Software de análise de confiabilidade)

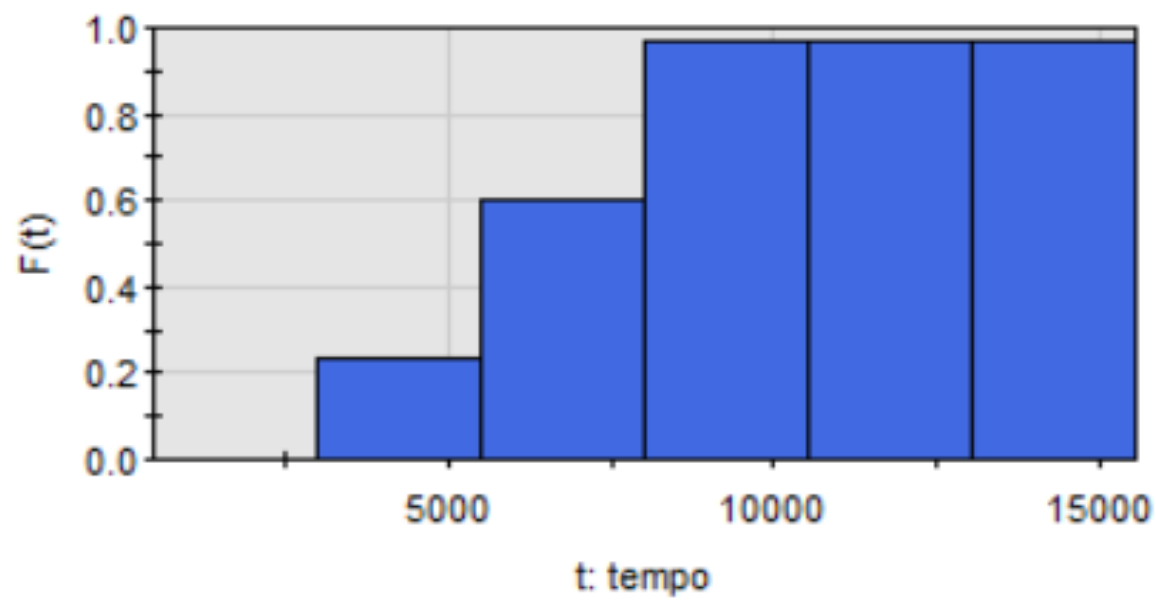

FIGURA 3 - Gráfico de barras de $\mathrm{F}(\mathrm{t})$ do estabilizador da aeronave

FONTE: Proconf (Software de análise de confiabilidade)

Foi proposta uma simplificação do sistema total da aeronave, tendo em vista a FIGURA 1, a fim de facilitar a demonstração de cálculo para $\mathbf{R}_{\mathrm{s}}$. Essa simplificação exibe a associação de componentes relacionados ao sistema de motores da aeronave, como mostrado pela equação (5), sendo $m$ os subsistemas $A, B, C$ e $D$, cuja identificação numérica $n$ é $1,2,3$ e 4 respectivamente.

$$
R_{m}=\left(R_{\text {motor } n}\right) x\left(R_{\text {combustível } n}\right) \times\left(R_{A \& R n}\right) \times\left(R_{\text {hélice } n}\right)
$$


Logo, efetuando-se os produtos, obtêm-se os seguintes resultados:

$$
R_{A}=R_{B}=R_{C}=R_{D}=R=0,9998584
$$

Ainda, com o objetivo de facilitar o cálculo de $\mathrm{R}_{\mathrm{s}}$, foi relacionado a um único subsistema $E$ o conjunto de componentes próprios do sistema de controles da aeronave, mostrado pela equação (6).

$$
\begin{gathered}
R_{E}=\left(R_{\text {direcional }}\right) \times\left(R_{\text {longitudinal }}\right) \times\left(R_{\text {lateral }}\right) \times\left(R_{\text {flaps }}\right) \times\left(R_{\text {estabilizador }}\right) \times\left(R_{\text {hidráulico }}\right) \\
x\left(R_{\text {trem de pouso }}\right) x\left(R_{\text {leme de direção }}\right) x\left(R_{\text {asa } 1}\right) x\left(R_{\text {asa } 2}\right)
\end{gathered}
$$

Logo,

$$
R_{E}=0,9821912
$$

Como especificado na seção 2.3, para a realização do cálculo de Rs, será utilizado o método do tie set. Para tanto, os tie set's mínimos obtidos são:

$$
\begin{aligned}
& T 1=\text { caminho }[A B E] \\
& T 2=\text { caminho }[A D E] \\
& T 3=\text { caminho }[C D E] \\
& T 4=\text { caminho }[C B E]
\end{aligned}
$$

Efetuando-se o devido cálculo para a união dos quatro eventos associados aos tie set's mínimos, obtêm-se e expressão matemática exibida pela equação (7). Ao substituir os valores de confiabilidade correspondentes a cada um dos subsistemas $A, B, C, D$ e $E$, a confiabilidade do sistema total $\left(\mathrm{R}_{\mathrm{s}}\right)$ pode ser obtida. 


$$
R_{s}=4\left(R^{2} R_{E}\right)-4\left(R^{3} R_{E}\right)+\left(R^{4} R_{E}\right)
$$

Logo,

$$
R_{s}=0,9821912
$$

\subsection{Cálculo da medida de Importância de Birnbaum}

Como especificado na seção 2.5, com o objetivo de detectar os componentes críticos do sistema, a medida de Importância de Birnbaum foi adotada.

Ao aplicar-se a equação (4), assumindo $i=A$, têm-se:

$$
I^{B}(A / t)=2\left(R R_{E}\right)-3\left(R^{2} R_{E}\right)+\left(R^{3} R_{E}\right)
$$

Visto que $R_{A}=R_{B}=R_{C}=R_{D}$,

Logo,

$$
I^{B}(A / t)=I^{B}(B / t)=I^{B}(C / t)=I^{B}(D / t)=1,390627649 \times 10^{-4}
$$

Da mesma forma, ao aplicar-se a equação (4), assumindo $i=E$, têm-se:

$$
I^{B}(E / t)=4 R^{2}-4 R^{3}+R^{4}
$$


Da equação (9) obtêm-se o seguinte resultado:

$$
I^{B}(E / t)=0,999999959
$$

Dessa forma,

$$
I^{B}(A, B, C, D / t)<I^{B}(E / t)
$$

Analisando os resultados obtidos da equação (8) e equação (9), é possível verificar que a importância dos componentes $A, B, C$ e $D$, é a mesma (se for considerado apenas os valores de confiabilidade dos mesmos, visto que a confiabilidade destes é a mesma). Nesse sentido, somente a posição dos componentes no sistema define sua importância (FOGLIATTO, 2006). Assim sendo, os componentes $C$ e $D$, para os quais não há caminho alternativo, são os de maior importância (ver FIGURA 1). Os componentes que oferecem um caminho alternativo para os demais, no caso $A$ e $B$, servem como ponte de conexão para $C, D$ e $E$, seguindo no ranking de importância, como mostrado na TABELA 3.

TABELA 3 - Ranking de Importância de Birnbaum FONTE: Os autores (2019)

\begin{tabular}{|c|c|}
\hline Componente & Birnbaum \\
\hline C & $1^{\circ}$ \\
\hline D & $1^{\circ}$ \\
\hline A & $2^{\circ}$ \\
\hline B & $2^{\circ}$ \\
\hline
\end{tabular}


Ao considerar-se o sistema de controles da aeronave, estruturado em série puro (reduzido simplificadamente ao componente $E$ ), é conhecido que o componente mais fraco de um arranjo em série é o mais importante (FOGLIATTO, 2006). Nesse sentido, o estabilizador é considerado o componente crítico do sistema de controles da aeronave, uma vez que seu valor de confiabilidade é o menor comparado aos demais. Ao atribuir o mesmo raciocínio para o fragmento em série puro do sistema de motores que envolve os componentes: motor, combustível, ventilação e aquecimento, e por fim, hélice, para $A, B, C$ e $D$, a unidade de menor confiabilidade é o combustível (sistema responsável pela injeção de combustível), sendo portanto, para o sistema de motores da aeronave, o componente mais crítico.

\subsection{FMEA}

Através de um brainstorming, a análise da FMEA foi realizada como é mostrado na FIGURA 4. 


\begin{tabular}{|c|c|c|c|c|c|c|c|}
\hline 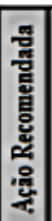 & 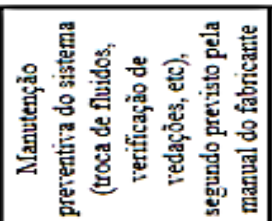 & & 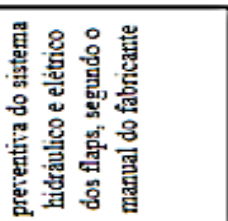 & 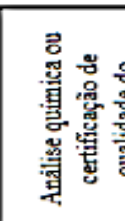 & 苟 & 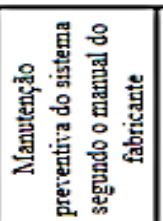 & 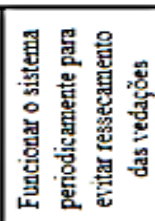 \\
\hline 总 & $\approx$ & 웅 & 요 & ๘ & 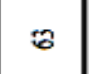 & $\therefore$ & 6 \\
\hline 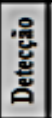 & - & - & - & - & - & - & - \\
\hline 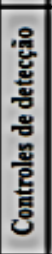 & 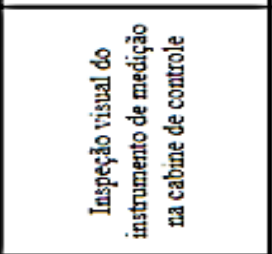 & & 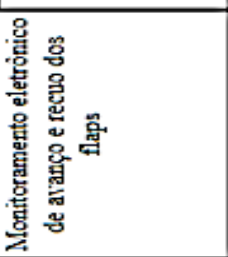 & 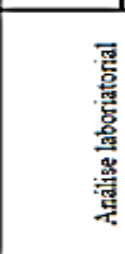 & & 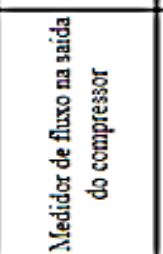 & 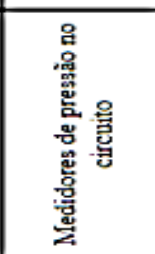 \\
\hline 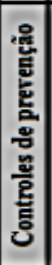 & 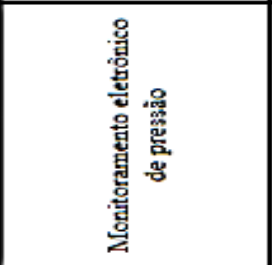 & 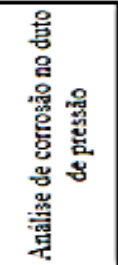 & 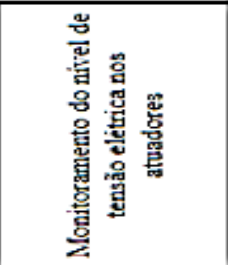 & 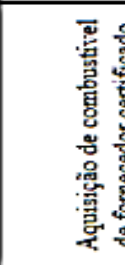 & & 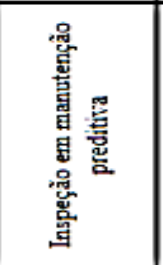 & 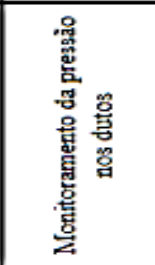 \\
\hline 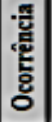 & $\infty$ & r & n & n & $n$ & r & $r$ \\
\hline 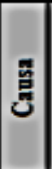 & $\begin{array}{l}\text { 을 } \\
\text { 量 } \\
\text { 鄫 } \\
\text { 喜 }\end{array}$ & 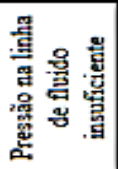 & 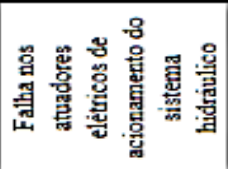 & 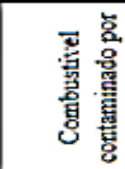 & & 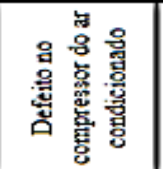 & 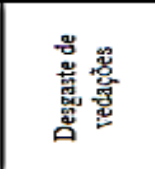 \\
\hline 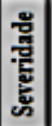 & $a$ & 으 & 으 & $a$ & $a$ & $n$ & $a$ \\
\hline 这 & 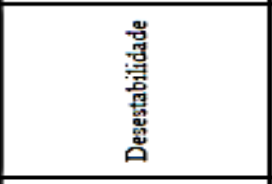 & & 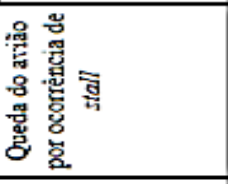 & 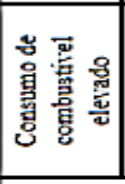 & 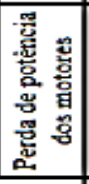 & 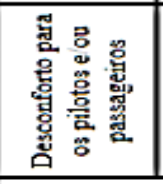 & 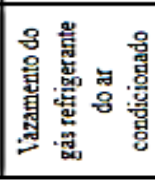 \\
\hline 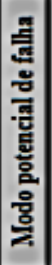 & 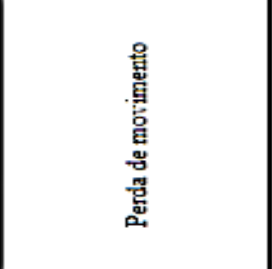 & & 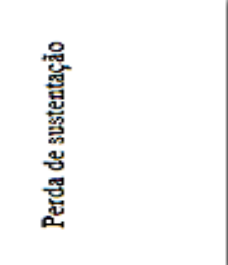 & 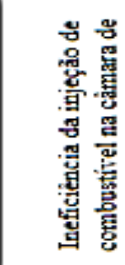 & & & 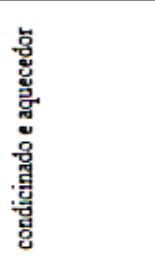 \\
\hline 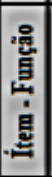 & 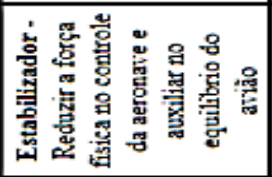 & 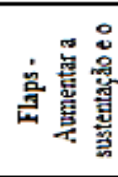 & 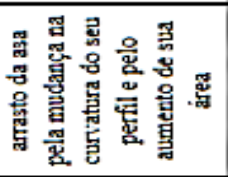 & 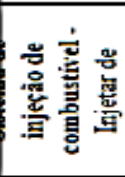 & 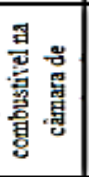 & 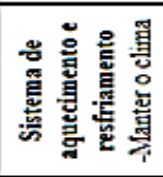 & 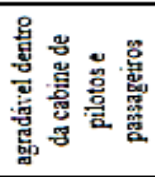 \\
\hline
\end{tabular}

FIGURA 4 - Análise da FMEA dos componentes críticos da aeronave FONTE: Os autores (2019) 
A título de explicação da FMEA, a linha mais importante é aquela que apresenta o maior Número de Prioridade de Risco (NPR). Isto significa que o componente estabilizador é o mais crítico dentre os analisados, cujo NPR é igual a 72, conforme a FIGURA 4. Para efeito de análise, os critérios utilizados para o cálculo do NPR são: (i) probabilidade de ocorrência de falha; (ii) possibilidade de detecção do modo de falha e (iii) grau de severidade do efeito da falha (FOGIATTO, 2006).

Para o cálculo do índice de ocorrência foi levado em consideração a probabilidade de falha do componente, em que Fogliatto (2006) apresenta uma sugestão de critério de avaliação quantitativa com base na taxa de falha, para dessa forma, determinar a classificação em que o componente em questão se enquadra. Ainda o autor apresenta uma sugestão de critério de caráter qualitativo de detecção e severidade, o qual foi utilizado como base neste presente trabalho.

\subsection{FTA}

Segundo os resultados de criticidade obtidos pela medida de Importância de Birnbaum e estudo da FMEA (ver seção 4.2 e 4.3), o estudo da FTA foi implementado somente para o componente do estabilizador (compensador) da aeronave, para fins de simplificação de demonstração, como mostrado na FIGURA 5.

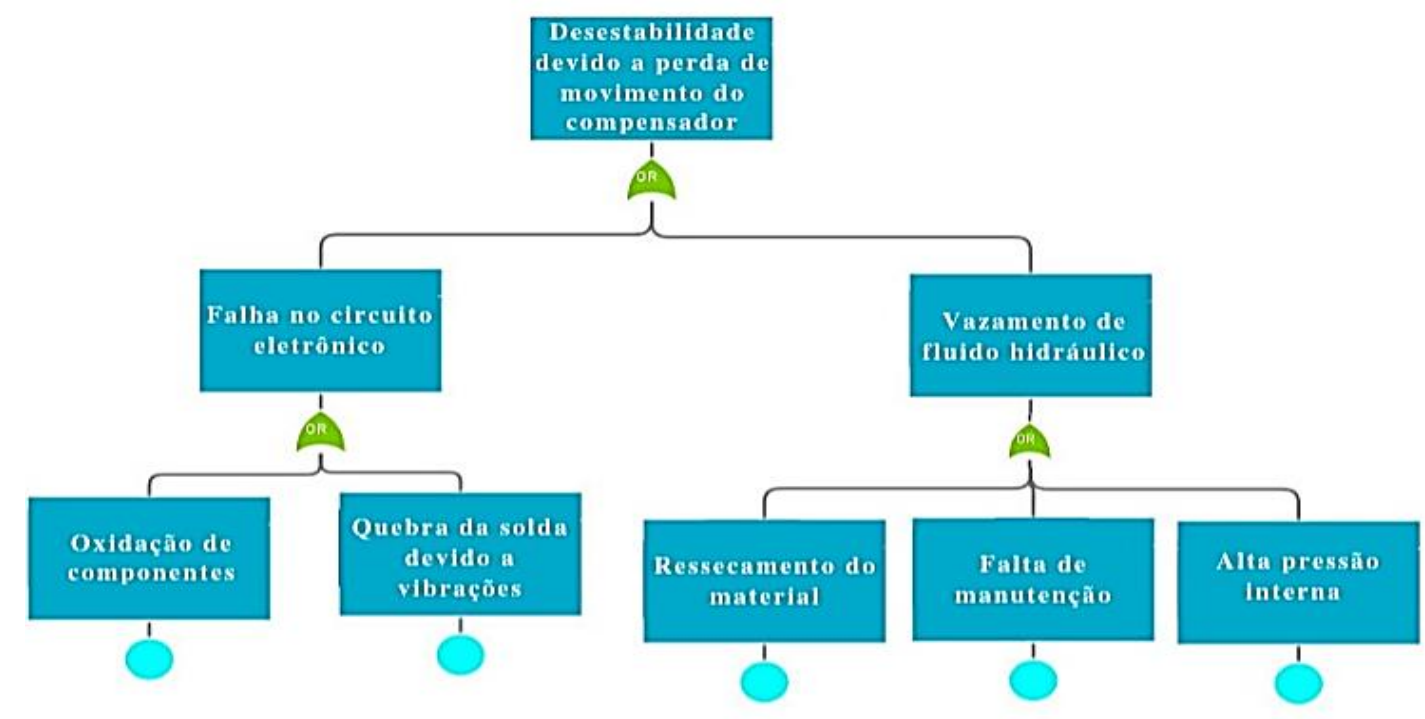

FIGURA 5 - FTA para o estabilizador da aeronave

FONTE: Os autores (2019) 
Partindo do evento topo 'desestabilidade devido à perda de movimento do compensador', duas falhas intermediárias foram levantadas como críticas: (i) falha no circuito eletrônico ou (ii) vazamento de fluido hidráulico. A primeira está sujeita a duas possíveis causas raízes independentes, em que a ocorrência de apenas uma destas já acarreta na falha do evento intermediário e consequentemente do evento topo. Já a segunda falha intermediária, é caracterizada por outras três falhas raízes independentes que seguem o mesmo raciocínio exposto anteriormente.

\section{CONCLUSÃO}

O presente artigo procurou apresentar, de forma sucinta, os principais procedimentos relacionados a realização de análise de confiabilidade em sistemas estruturais complexos, utilizando como estudo de caso uma aeronave quadrimotora. A metodologia aplicada visou estimar a confiabilidade associada ao sistema total. Para análise de falha por meio da FMEA e FTA, foram considerados os componentes mais relevantes do sistema, por meio da análise de Medida de Importância de Birnbaum. Os principais métodos foram exibidos e sua aplicabilidade foi demonstrada através do arranjo estrutural da aeronave considerada e dos dados de confiabilidade dos componentes disponibilizados por meio de levantamento bibliográfico.

Os métodos utilizados permitem a realização de programas de melhoria contínua, bem como viabiliza um maior acesso por parte da equipe de engenheiros e técnicos ao verdadeiro cenário comportamental das falhas do sistema de engenharia, em termos de confiabilidade, criticidade, causas raízes, efeitos, riscos e ações para o tratamento dessas falhas.

\section{REFERÊNCIAS}

DODSON, H. S. B. Accelerated testing: apractitioner's guide to accelerated and reliability testing. [S.1.]: SAE Internacional, p.19, 2006.

ELSAYED, E.A. Reliability Engineering. Nova York: Prentice-Hall, p.737, 1996.

FOGLIATTO, J. R. F. Confiabilidade e manutenção industrial. [S.1.]: Elsevier, p.87-96, 2006.

LEEMIS, L. Reliability: probabilistic models and statistical methods. Nova York: Prentice- 
Hall, p.384, 1995.

LENZ, M; Rhodin, J. Reliability calculations for complex systems. Semantic Scholar, p.44, 2011.

KNIGHT, C.R. Four decades of reliability progress. Proceedings of the Annual Reliability and Maintainability Symposium. [S.1.]: IEEE Reliability Society, p.156-159, 1991.

SMITH, D. J. Reliability, maintainability, and risk: practical methods for engineers. 6. ed. [S.1.]: Butterworth-Heinemann Ltd 1993, p.118, 2001.

RAUSAND, M.; HØYLAND, A. System Reliability Theory: Models, Statistical Methods, and Applications. 2. ed. Nova York: John Wiley, p.183-193, 2003.

PINHEIRO, J., et al. Probabilidade e estatística: quantificando a incerteza. Elsevier Brasil, p.150, 2013.

BIROLINI, A. Reliability Engineering: Theory and Practice. 5. ed. [S.1.]: Springer, p.72, 2007. PETTIT, D., Turnbull, A. and Roelant, H.A. General aviation aircraft reliability study. p.24-27, 2001. 